\title{
Setting the Trend for the Alternatives Movement in India: The Second National Conference of the Society for Alternatives to Animal Experiments-India
}

\author{
doi:10.14573/altex.2003183
}

\begin{abstract}
Introduction
The Second National Conference of the Society for Alternatives to Animal Experiments (NCAAE-2019) was organized at National Center for Biopharmaceuticals (NFB), Guru Nanak Khalsa College, Mumbai, India, with Dr Pratiksha Palahe as the Organizing Secretary, on December 13-14, 2019. The theme Trends in the Science of Alternatives offered a platform for understanding the challenges in promoting alternatives to animal testing in India and other Asian countries. The conference with over 100 participants from all over India was successfully conducted with talks by invited speakers from India and abroad and by mid-career scientists. Posters were displayed and oral presentations were made by students. Speakers and delegates representing industry and regulators took part, so there was exchange of ideas between academics, animal protectionists, regulators and CROs. Pre- and post-conference workshops offered intensive hands-on training on different aspects of alternatives.
\end{abstract}

\section{Inauguration}

Dr Ganapati D. Yadav, Former Vice Chancellor, Institute for Chemical Technology, Mumbai, inaugurated the conference. While recalling the contribution of animal experiments to drug discovery and risk assessment, he emphasized the need to catch up with the trend in research methodologies, especially non-animal methods. "Scientists from biological sciences should work collaboratively, invoking artificial intelligence technology, and develop more alternatives for use in place of animal experiments," he said. Dr Kiran Mangaonkar, Principal, G.N. Khalsa College, welcomed the gathering. Dr Yogendra K. Gupta (President, SAAE-I) and Dr Mohammad A. Akbarsha (General Secretary, SAAE-I) briefly recalled the history of SAAE-I, its objectives and progress so far, and the objectives of the conference. Dr Pratiksha Palahe, Organizing Secretary, presented the program. A memorandum of understanding (MoU) was signed between Japanese Society for Alternatives to Animal Experiments (JSAAE) (represented by Dr Yasuyuki Sakai, President) and SAAE-I (represented by Dr Gupta). Dr Rubina Bose, Deputy Drug Controller General (West Zone, India) and Dr Smita Mahale, Director, National Institute of Research in Reproductive Health (under Indian Council for Medical Research), Mumbai, the guests of honor, offered felicitation. Ms. Shalmali Pendse, the Co-organizing Secretary, thanked all contributors.

\section{Scientific Lectures}

Dr Thomas Hartung, Director, Center for Alternatives to Animal Testing (CAAT) at Johns Hopkins University, Baltimore, MD, USA, delivered a virtual plenary lecture on "Perspectives of non-animal models". He stated that while the limitations of animal-based toxicology to predict human health issues are being increasingly recognized, shortcomings of traditional (human) cell culture are also evident. Issues around these include cell identity, differentiation, genetic stability and mycoplasma infection as well as non-homeostatic and non-physiological culture conditions. Large toxicological databases ("big data") and data-mining technologies ("artificial intelligence") now allow predictive computational approaches on a new scale, for example, using automated read-across (RASAR, i.e., read-across-based structure activity relationships). At the same time, the combination of cell culture with bioengineering has led to more organotypic cell culture technologies, such as 3D culture, human stem cell-derived systems, perfusion, co-cultures, combinations with scaffolds and sensors, etc. Increasingly, these approaches are combined to organ-on-chip or even multi-organ human-on-chip solutions. By recreating organ architecture and functionality and homeostasis of the cell environment, these models mirror the physiological situation more closely. The example of the human iPSC-derived mini-brain developed at CAAT illustrates this claim. Combined with an increased mechanistic base of reasoning (e.g., adverse outcome pathway (AOP) concepts, integrated testing strategies (ITS), and evidence-based methods of data evaluation and integration), these are revolutionary changes in how we can assess the biological effects of substances.

Dr Christian Pellevoisin, Scientific Director, EPISKIN Academy, Lyon, France, talked about "Medical devices: ISO 10993 series moves to alternative methods". He said that reconstructed human epidermis (RhE) allows validated full or partial replacement of different in vivo assays, is integrated in OECD Test Guidelines, and is used in screening and in support of weight of evidence approaches. Recently, RhE has been adapted to testing biocompatibility of medical devices, monitoring devices, and instruments used in diagnostics. The project conducted under Workgroup 8 (WG8) of the Technical Committee 194 (TC194) for biocompatibility of medical devices aims to replace the in vivo Draize test by an in vitro method using RhE models. The recent outcome of the round robin study with two RhE models in 17 lab- 
oratories has led to a new standard (ISO 10993-23) that will be released in 2020. At the same time, several projects based on human reconstructed tissues for in vitro eye irritation, vaginal irritation or skin sensitization by medical devices are ongoing. The translation of these in vitro approaches to updated or new ISO 10993 standards will provide adapted non-animal approaches for risk assessment of medical devices.

Dr Kwang-Mahn Kim, Department of Dental Biomaterials \& Bioengineering, Yonsei University College of Dentistry, Seoul, Republic of Korea, spoke on "Alternative methods of biocompatibility test for medical devices used in dentistry", in which he focused on biocompatibility testing for dental biomaterials. The dentin barrier test, murine local lymph node assay (LLNA), RhE test for skin irritation, and other biomimetic cytotoxicity tests are being assessed for biocompatibility testing. Dentin substitute materials, e.g., polyurethane discs, have been developed for the dentin barrier test and a test protocol for an in vitro oral mucosa irritation test using 3D culture of oral epithelial tissue has been defined. Additionally, the ISO TC 194/WG 8 round robin study for skin irritation test methods for medical devices will take testing of medical devices to the next level.

Dr Albert P. Li, President, CEO and Co-founder, In Vitro ADMET Laboratories LLC, Columbia, MD and Malden, MA, USA, addressed "In vitro human experimental systems for the evaluation of xenobiotic properties: hepatocytes, enterocytes and IdMOC'. According to him, experimentation with laboratory animals does not always provide data that are translatable to humans because of species differences. This is particularly important in the evaluation of xenobiotic toxicity. The lecture addressed species differences in xenobiotic metabolism and human in vitro experimental systems that can replace in vivo animal experiments. He elaborated upon the applications of human hepatocytes, enterocytes, and the integrated multiple organ co-culture (IdMOC) system in the evaluation of xenobiotic metabolism and toxicity.

Dr Jayasujatha Vethamanickam, Safety Scientist, Safety and Environmental Assurance Centre, Unilever R\&D, UK, in her talk on "Non-animal approaches for cosmetic safety assessment: A case study" stated that several countries, including India, have banned animal testing of cosmetics and their ingredients. The Bureau of Indian Standards (BIS) is currently updating the Methods of Tests for Safety Evaluation of Cosmetics to include more non-animal methods that have been accepted in other countries. As recently outlined by the International Cooperation on Cosmetics Regulation (ICCR), the key principle is that next generation risk assessments (NGRA) for cosmetic safety should be exposure-led and hypothesis-driven. A key metric in this case is the point-of-departure ( $\mathrm{PoD})$, the lowest concentration at which biological responses can be detected within a given set of non-animal assays. The ICCR principles were applied to a hypothetical safety assessment of $0.1 \%$ coumarin in face cream. Exposure calculations using specific consumer habits data were used to build a physiologically-based kinetic model and a battery of in vitro assays was used to determine PoDs for a variety of biological effects including genotoxicity, immunomodulatory effects and activity against a panel of biological targets. The PoDs from the in vitro assays were plotted against the calculated in vivo exposure to determine a margin of safety (MoS). From these results, it was concluded that coumarin is not genotoxic, does not bind to the 44 receptors tested or show any immunomodulatory effect. The case study demonstrated the importance of integrating exposure science with computational modeling and in vitro bioactivity data as the basis of non-animal safety assessments.

Dr Eui-Bae Jeung, Laboratory of Veterinary Biochemistry and Molecular Biology, College of Veterinary Medicine, Chungbuk National University, Republic of Korea, dwelt on "Alternative developmental and neurological test based on stem cell". The developmental toxicity test method (termed EBT) measuring the area of embryoid bodies (EBs) has undergone a validation study using a new predictive model. 61 chemicals were predicted with an average accuracy above $80 \%$. The high-throughput developmental neurotoxicity test (DNT) method measuring cell viability and inhibition of neuronal differentiation based on GFP intensity using Sox1-GFP stem cells was optimized and challenged with developmental neurotoxicants. Together, the results suggest that EBT and DNT can be useful for evaluation of the toxicity of new chemicals or candidate drugs. A close collaboration between Korean and Indian research groups in the field of alternatives was proposed; the group has already signed a MoU for collaborative research efforts with the Department of Medical Elementology \& Toxicology, Jamia Hamdard, New Delhi, India.

Dr Dipti Kapoor, representing People for the Ethical Treatment of Animals (PETA)-India, New Delhi, spoke on "The need for non-animal recombinant antitoxins". Most of the currently available antitoxins are harvested from the blood of immunized animals. This process can cause local and systemic adverse effects, including injection-site edema, thrombosis, phlebitis, abscesses, fistulas, and fibrosis. The resulting drugs derived from animal serum have well-documented safety issues including hypersensitivity reactions and serum sickness, and maintenance of reliable stockpiles for quick transport to patients in need of treatment is challenging. Several recombinant antibody-based therapeutics have been developed and approved for clinical use, and more are in the pipeline.

Dr Brinda Poojary, from the Research \& Toxicology Department of Humane Society International, Hyderabad, India, spoke about "Vaccine global regulatory harmonization". Vaccines are among the greatest public health achievements. They have helped eradicate certain diseases and have increased life expectancy. The talk provided an overview of the role of animal models in vaccine production, the current scenario of working towards elimination of redundant tests, and the way forward towards alternative methods in the regulatory and industry sectors.

Dr Abhay Deshpande, Director, Global Innovation and Strategy, Jai Research Foundation, Vapi, Gujarat, India, delivered a lecture on "Novel alternative methods: Global efforts bearing fruits". He spoke on the different contributions of research groups, agencies and organizations, industry and regulators to the development and acceptance of new approach methods (NAM).

Dr Barry Hardy, President, Open Tox Association, and CEO at Edelweiss Connect Inc., GmbH, Basel, Switzerland, gave a virtual presentation on "International collaborations advancing the development and applications of new alternative methods in predictive toxicology and risk assessment". He introduced OpenRiskNet as an open e-infrastructure providing resources and ser- 
vices to a variety of communities requiring risk assessment, including chemicals, cosmetic ingredients, therapeutic agents and nanomaterials. He also discussed the challenges of proposed activities to bring people and diverse resources to the same table to accelerate international collaboration that will support conversation and knowledge activities, leading to strong and rewarding outcomes.

Dr Suresh Poosala, Director, Reagene Bio-Sciences, Hyderabad, India, delivered a talk on " 3 D bio-printing to promote drug discovery and replace animals in research". The 3D bio-platform aims to de-risk clinical and product failures early on by improving the translatability of many assays as a cost-effective and highly predictive alternative to testing in animals. Functional units that reflect human pathophysiology are being generated. The translational assay platform may be custom-designed for specific needs to address targeted questions.

Dr Sameer Jhadav, Department of Chemical Engineering, Indian Institute of Technology, Mumbai, India, spoke on "Bioassay for characterizing sperm motility". Two bio-assays to measure sperm motion were introduced. The first involves a microfluidic device to measure the chemotactic response of sperm, the second characterizes the motion of sperm having immobilized heads.

Dr Anne Gourmelon, Principal Administrator - Test Guidelines Programme, Environment, Health and Safety Division, Environment Directorate, Organization for Economic Cooperation and Development (OECD), delivered a virtual talk on "OECD works on transition to combinations and defined approaches for complex hazard endpoints". The OECD develops test guidelines that enable industries that follow GLP principles to generate data that are accepted across member countries, including India, in the Mutual Acceptance of Data system. In the last decade, OECD has adopted numerous non-animal methods for simple toxicities. For more complex and systemic toxicities, a number of projects are now directed towards the combination of in vitro methods that represent key events in AOPs and in silico approaches.

Dr Yasuyuki Sakai, Department of Chemical System Engineering and Department of Bioengineering, Graduate School of Engineering, University of Tokyo, Japan, and President of Japanese Society for Alternatives to Animal Experiments (JSAAE), spoke on "Latest activities and future directions of JSAAE for Asian cooperation toward $3 R$ ". The JSAAE promotes 3 Rs research in Japan through a wide variety of activities such as hosting of the $6^{\text {th }}$ World Congress on Alternatives and Animal Use in Life Sciences in Tokyo (2007) and contributing to the development of guidelines in cooperation with Japanese Center for the Validation of Alternative Methods (JaCVAM). JSAAE now has MoU with counterpart societies in Korea, EU and USA and is planning to set up MoU with Chinese counterparts. To strengthen communication among Asian communities, the Society is planning to set up an Asian Consortium of Alternatives to Animal Experiments.

Dr Hajime Kojima, representing JaCVAM, National Institute of Health Sciences (NIHS), Tokyo, Japan, explained how JaCVAM has been instrumental in promoting the development and evaluation of alternatives to test methods in Japan. JaCVAM's mission is to promote the use of alternative methods to animal testing in regulatory studies while ensuring protection of the public by assessing safety of chemicals and other materials. JaCVAM plays an active role in the application and approval process for permission to manufacture and sell pharmaceuticals and other products as well as in revising standards for cosmetic products. JaCVAM participates in International Cooperation on Alternative Test Methods (ICATM) activities, under which it has organized a number of international validation studies that have led to the issuance of fourteen test guidelines and one guidance document by the OECD. Dr Kojima recommended the establishment of a center for validation in India.

Dr Sheikh Raisuddin, Department of Medical Elementology \& Toxicology, Jamia Hamdard (Institute of Eminence \& Deemed University), New Delhi, India, addressed "School level curriculum intervention for awareness about animal welfare and alternatives". Under the initiative of PETA, the National Council of Educational Research and Training (NCERT) and Central Board of Secondary Education (CBSE) have agreed to incorporate a humane-education program in school curricula. STRAW (Stray Relief and Animal Welfare) India contributes content relating to empathy for people, compassion for animals, and care for the environment to school textbooks and teachers' manuals. Animations and computer-aided dissection have taken firm root in the school education system in India. It also has been agreed by the school boards to phase out use of leather shoes as part of school uniform. In higher education, the University Grants Commission (UGC), Medical Council of India (MCI), Pharmacy Council of India (PCI), Veterinary Council of India (VCI), and National Council of Teacher Education (NCTE) also support animal welfare and alternatives in their curricula. In 2014, UGC issued directives to discontinue dissection and animal experimentation in laboratory courses at undergraduate and post-graduate levels. In his talk, Dr Raisuddin applauded the role of Bombay Society for the Prevention of Cruelty to Animals (BSPCA) including the Bai Sakarbai Dinshaw Petit Hospital for Animals.

\section{Presentations by mid-career scientists}

Dr Shilpy Sharma, from Department of Biotechnology, Savitribai Phule Pune University, Pune, talked on "Alternatives to animal experiments for studying type 2 diabetes mellitus and associated metabolic disorders". She reported a modality for rapid screening of compounds for protection of pancreatic $\beta$-cells against human islet amyloid polypeptide (hIAPP) using recombinant INS-1E cells and on the generation of functional human pancreatic islets using dental pulp-derived stem cells to study the insults caused by high glucose, lipid and hIAPP. Dr Syed Ziaur Rahman, Faculty In-Charge, Alternatives to Animal Experimentation Lab, Department of Pharmacology, Jawaharlal Nehru Medical College, Aligarh Muslim University, Aligarh, India, shared his experience on "Teaching and learning of experimental pharmacology by alternatives". In his institute, animal experiments have been taught using computer-based simulators since 2006. Undergraduate students learn the basic concepts of pharmacology with the help of around 53 software-based pharmacology experiment teaching modules. Dr Banappa S. Unger, Pharmacology and Toxicology Division, National Institute for Traditional Medicine, under Indian Council for Medical Research (ICMR-NITM), Belagavi, Karnataka, India, shared his 
team's success in using zebrafish as an alternative animal model to assess the side effects of atypical antipsychotic medication for psychiatric disorders, especially olanzapine. Dr Lekha Divya, Department of Zoology, Central University of Kerala, Kasaragod, Kerala, India, shared her experience with the red ant as an alternative model for toxicological evaluation of nanomaterials and nanowastes in non-target organisms.

Dr Sreejith P. Panicker, Department of Zoology, University of Kerala, Thiruvananthapuram, India, highlighted the conduct and outcome of the pre-conference workshops on alternative animal models organized under the aegis of SAAE-I (zebra fish and echinoderms to study tissue regeneration, conducted by Dr Mohammed Idris from Centre for Cellular and Molecular Biology (CCMB), Hyderabad, India, and his team, on November 25-29, 2019) and in vitro toxicity evaluation adopting integrated discrete multiple organ co-culture (IdMOC) conducted on December 9-11, 2019, by Dr Albert P. Li, In Vitro ADMET Laboratories Inc, Columbia, MD, USA). Others associated as speakers/ trainers were Dr Sheikh Raisuddin, Department of Medical Elementology \& Toxicology, Jamia Hamdard (Deemed University), New-Delhi (Topic: Potential of invertebrates as test organisms); Dr Mohammad A. Akbarsha, National College (Autonomous), Tiruchirappalli, India (Topics: i) Animal models of lesser sentience; and ii) Animal cell culture techniques and tools); Dr Prabha D. Nair, from Sree Chitra Tirunal Institute for Medical Sciences \& Technology, Thiruvananthapuram, India (Topic: Science of tissue regeneration- the case of the cartilage); and Dr Anil Kumar, from Sree Chitra Tirunal Institute for Medical Sciences \& Technology, Thiruvananthapuram, India (Topic: 3D Bioprinting using multiple cell types).

\section{Preconference and Post-Conference Workshops}

The pre-conference workshops conducted at Department of Zoology, University of Kerala, Thiruvananthapuram, India, have been covered vide supra. Another preconference workshop on Adverse Outcome Pathways (AOP), a joint venture of Centre for Predictive Human Model Systems and Edelweiss Connect, Switzerland, and Humane Society International, India, was hosted by the National Facility for Biopharmaceuticals, Mumbai, India, on December 12, 2019. Around 100 participants attended. Dr Surat Parvatam, Research Associate, Centre for Predictive Human Model Systems, AIC-CCMB, HIS-India, and Dr Tatyana Doktrova, Senior Toxicologist, Edelweiss Connect, Switzerland, were the speakers/trainers for the session. Dr Brinda Poojary, Campaign Manager, Research and Toxicology Department, HIS-India, spoke about the call for proposals under the theme of AOP. Dr Pratiksha Palahe, Head, R\&D, National Facility for Biopharmaceuticals, Mumbai, was the Organizing Secretary.

Post-conference workshops "Alternatives and skin irritation test according to OECD TG-439 using reconstructed human epidermis (RhE)" were held on December 15-16, 2019 at National Facility for Biopharmaceuticals, Mumbai, India, and on December 18-19, 2019, at Department of Elementology \& Toxicology, Jamia Hamdard, New Delhi. Each was a joint venture of the respective institute with EPISKIN Academy, Lyon, France, and SAAE-I. Dr Pratiksha Palahe and Dr Sheikh Raisuddin, respectively, extended the laboratory support, and Dr Christian Pellevoisin, Scientific Director, Episkin Academy, was the key resource person in both events.

\section{Poster and oral presentation competition}

A research poster presentation competition was held. Eight posters were selected for detailed oral presentation, of which three received oral presentation gold medals and three received poster presentation gold medals, and the remaining two were conferred consolation prizes. Travel cash awards were presented to Dr Shilpy Sharma and Dr Lekha Divya.

\section{Conclusion}

Overall, the second SAAE-India conference generated invigorated and enthusiastic response from stakeholders in India and abroad. It is hoped that the conferences and workshops in the future will be organized with more scope for presentations by all stakeholders, especially the young researchers.

\section{Acknowledgements}

The conference was supported financially by Science and Engineering Research Board (SERB), Department of Science and Technology, Government of India, New Delhi, Humane Society International, Doerenkamp-Zbinden Foundation, PETA-India, HiMedia Laboratories Pvt Ltd., Thermo Scientific, Jai Research Foundation, and Episkin Academy. The awards were sponsored by Episkin Academy- Loreal combine, France. Open Tox and Edelweiss Connect, Switzerland also collaborated in the endeavor. The National Centre for Alternatives to Animal Experiments, Bharathidasan University, Tiruchirappalli, India, was an academic partner. Additional academic support came from Dr Abhijit Majumder, Indian Institute of Technology, Mumbai, Dr Deepak Modi, National Institute for Research in Reproductive Health, Mumbai, and Dr Prajakta Dandekar, Institute for Chemical Technology, Mumbai. Great administrative and facility supports were availed from Sardar Gurinder Singh Bawa, Chairman, G.N. Khalsa College Management Committee, and Dr Kiran Mangaonkar, Principal, Guru Nanak Khalsa College. The technical support of Mr Dattaray Sawant and Mr. Tuskar Kamble, NFB, Mumbai, is gratefully acknowledged.

\section{Pratiksha Palahe ${ }^{1}$, Shalmali Pendse ${ }^{1}$,Vinal Pardhi ${ }^{1}$, Vikas Jha ${ }^{l}$, Valencia D'souza', Sreejith P. Panicker ${ }^{2}$, Sheikh Raisuddin ${ }^{3,5}$ and Mohammad A. Akbarsha ${ }^{4,5}$}

${ }^{1}$ National Facility for Biopharmaceuticals, G.N. Khalsa College, Matunga, Mumbai, India; ${ }^{2}$ Department of Zoology, University of Kerala, Kariavattom, Thiruvananthapuram, India; ${ }^{3}$ Department of Medical Elementology and Toxicology, Jamia Hamdard (Deemed University), New Delhi, India; ${ }^{4}$ National College (Autonomous), Tiruchirappalli, India;

${ }^{5}$ Society for Alternatives to Animal Experiments, Bharathidasan University, Tiruchirappalli, India 